\title{
CLINICAL PRACTICE Clinical Vignettes Risking Life and Limb: A Case of Spontaneous Diabetic Muscle Infarction (Diabetic Myonecrosis)
}

\author{
Jeremy Cumberledge, $M D^{7}$, Bharat Kumar, $\mathrm{MD}^{2}$, and David Rudy, $\mathrm{MD}^{7,3}$ \\ ${ }^{1}$ Department of Internal Medicine, University of Kentucky, Lexington, KY, USA; ${ }^{2}$ Department of Allergy and Immunology, Department of \\ Rheumatology, University of lowa, lowa City, IA, USA; ${ }^{3}$ Department of Internal Medicine, K521 Kentucky Clinic, Lexington, KY, USA.
}

Diabetic muscle infarction, also known as diabetic myonecrosis, is a microvascular complication of poorly controlled diabetes mellitus characterized by unilateral limb pain and swelling. Because these symptoms closely mimic cellulitis and thromboembolism, diagnosis is often delayed or missed altogether, leading to increased morbidity and inappropriate treatment. We describe a case of unilateral limb pain and swelling due to diabetic muscle infarction in a 36-year-old patient with poorly controlled diabetes mellitus. We also review the literature on the diagnosis, prognosis, and management of this underrecognized condition.

KEY WORDS: diabetes; muscle infarction; leg pain.

J Gen Intern Med 31(6):696-8

DOI: $10.1007 / \mathrm{s} 11606-015-3551-8$

(c) Society of General Internal Medicine 2015

\section{CASE}

\section{Introduction}

Leg pain is a common complaint among patients with diabetes mellitus, and is frequently assessed in the ambulatory, emergency, and inpatient settings. Due to its nonspecific presentation, diagnosis is hard to establish, often leading to delayed diagnosis, under-treatment, and excessive laboratory and radiographic testing. Diabetic muscle infarction is a rare cause of leg pain but is likely under-recognized in patients with complication of long-standing, poorly controlled diabetes mellitus. We present a case of a 31-year-old woman presenting with unilateral leg pain and swelling who was diagnosed with diabetic muscle infarction, and we review the diagnostic considerations as well as general prognosis and management of this condition. We also review the available literature and emphasize that physicians should keep a high index of suspicion for diabetic muscle infarction in patients with poorly controlled diabetes and limb pain.

\section{Case Presentation}

A 31-year-old woman with hypertension and type 2 diabetes mellitus complicated by retinopathy, peripheral neuropathy, gastroparesis, and nephropathy presented with left lateral calf pain that began at rest four days prior to admission. She denied antecedent trauma or prior episodes of leg pain. She described the pain as constant, worsened by exertion, partially improved

Published online December 7, 2015 by rest, and associated with localized 'knot-like' swelling for the past two days. She denied fevers, chills, and weakness of the affected leg.

On physical exam, vital signs were notable for temperature of $98^{\circ} \mathrm{F}$, blood pressure of $184 / 98 \mathrm{~mm} \mathrm{Hg}$, and heart rate of 78 bpm. Other significant findings included $2+$ pitting edema of the lower extremities bilaterally, with greater swelling of the left leg compared to the right. There was hair loss over the left lower leg, but no warmth or erythema. On palpation of the left lower leg there was noted tenderness. Dorsalis pedis pulses were $2+$ bilaterally. Patellar reflexes were hypoactive $(1+$ bilaterally), and strength of major muscle groups in both legs was $5 / 5$. Serum electrolytes, including phosphate and magnesium, were within normal limits; creatinine was elevated at $3.91 \mathrm{mg} / \mathrm{dL}$. There was slight leukocytosis (white blood count [WBC]: $12.2 \mathrm{k} / \mu \mathrm{l})$ and anemia $(\mathrm{Hg}: 9.2 \mathrm{~g} / \mathrm{dL})$. Systemic inflammatory markers were elevated (C-reactive protein [CRP]: $4.3 \mathrm{mg} / \mathrm{dL}$ [normal $0-0.9 \mathrm{mg} / \mathrm{dL}$ ] and erythrocyte sedimentation rate $[\mathrm{ESR}]>120 \mathrm{~mm} / \mathrm{h}$ [normal 0-20]), as were creatine kinase $[\mathrm{CK}](477 \mathrm{U} / \mathrm{L}$ [normal 38-176 U/L]) and hemoglobin A1c (14.4\%). Blood cultures were negative.

Bilateral lower-extremity duplex ultrasound revealed heterogeneous echotexture over the left foreleg musculature and subcutaneous edema without evidence of deep vein thrombosis (DVT). Due to preexisting kidney disease, MRI without contrast of the left lower extremity was performed. This revealed increased signal in the T1- and T2-weighted images within the lateral compartment and adjacent deep posterior compartment of the foreleg, concerning for compartment syndrome (Fig. 1). Due to the stability of the patient, empiric antibiotics were not given. Vascular Surgery was consulted and performed bedside manometry, which showed no evidence of increased compartment pressure. In order to rule out pyomyositis, a nuclear medicine abscess scan with $0.521 \mathrm{mCi}$ In-111-labeled autologous leukocytes was performed. There was no increased uptake of labeled leukocytes in the affected leg to suggest soft tissue infection. Orthopedic Surgery was consulted, and determined that the patient's condition fit well with diabetic muscle infarction. The MRI was then reviewed with the interpreting radiologist, and the increased signal in the T2-weighted images was thought to correlate well with a diabetic muscle infarction, and thus the diagnosis was made. The patient's insulin regimen was adjusted for better glycemic control while she was in the hospital, 


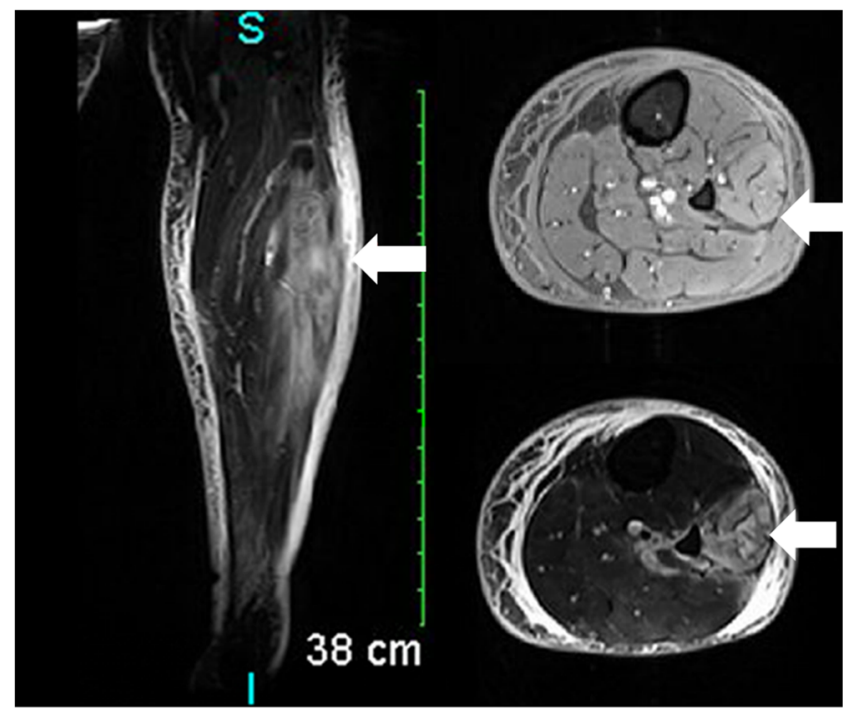

Fig. 1 T1-weighted coronal and T1- and T2-weighted axial MRI images of the patient's left lower extremity, significant for increased signal in the lateral and deep posterior compartments

and follow-up with an endocrinologist was arranged. The patient's leg pain had improved with analgesics throughout her stay, but was still present at the time of discharge. The patient was discharged with instructions for rest, low-dose aspirin, and medications for pain control.

\section{Discussion}

Diabetic muscle infarction, also known as diabetic myonecrosis, is a rare and likely under-recognized complication of diabetes mellitus. Despite its discovery approximately 50 years ago by Drs. Angervail and Stener, the pathophysiology and epidemiology remain largely unknown. $^{1}$ A systematic review in 2006 found 115 cases reported in the literature, but the actual incidence of diabetic muscle infarction is thought to be significantly higher. ${ }^{2}$ This review also suggested that diabetic muscle infarction occurs more frequently in middle-aged individuals (mean age of 43) and has a female predilection. ${ }^{2}$ The disease is frequently associated with severe microvascular complications in both type 1 and type 2 diabetes mellitus. Indeed, in patients ultimately diagnosed with diabetic muscle infarction, concomitant nephropathy, retinopathy, and neuropathy are seen in 71, 57, and $55 \%$ of cases, respectively. ${ }^{1}$ These epidemiologic data suggest that the condition may be mediated by arteriosclerotic phenomena; endothelial and platelet dysfunction have also been speculated to play a role in diabetic muscle infarction. ${ }^{1}$

Diabetic muscle infarction typically presents as suddenonset unilateral lower limb pain, most often in the thigh, although there may be bilateral involvement in approximately $30 \%$ of cases. ${ }^{2}$ Patients also frequently describe a mass-like area of induration in the affected limb. ${ }^{3}$ Systemic symptoms like fever are rarely observed in this condition, unlike in infectious processes, where fever predominates. ${ }^{4,5}$ Regardless, because the clinical findings of diabetic muscle infarction are nonspecific and often mimic other conditions such as DVT, pyomyositis, muscle abscess, muscle neoplasm, myonecrosis/necrotizing fasciitis, and hematoma, many cases are likely to go undiagnosed or may be treated inappropriately.

Compounding this diagnostic uncertainty is the lack of any discriminant laboratory findings. As in other causes of limb pain in diabetics, white blood cell count, creatinine kinase, and inflammatory markers may be elevated. Ultrasonography, if performed, typically shows abnormal linear echo-texture of the effected limb. ${ }^{6}$ CT findings in diabetic muscle infarction are less specific than those of MRI, but may show muscle enlargement with diminished attenuation, thickening of adjacent fascial planes and overlying skin, and increased subcutaneous attenuation. ${ }^{7}$ Recent reports have supported the use of MRI for diagnosis. Abnormal MRI findings, including high intensity in the affected muscle on fat-suppressed T-2 weighted images, carries a sensitivity close to $100 \%{ }^{8}$ Muscle biopsy is the gold standard for diagnosis, but may be avoided when classic history and MRI findings are present. If muscle biopsy is performed, pathology specimens typically show muscle necrosis and edema. Fibrin occlusion of arterioles and capillaries may also be seen. ${ }^{9}$

Tight glycemic control is the mainstay of treatment, as hyperglycemia has an effect on the remodeling of vasculature, platelet function, and coagulation factors, which may contribute to diabetic muscle infarction. Patients should also be encouraged to rest the affected limb in the acute period. In those patients who are not on antiplatelet therapies, aspirin is commonly prescribed, but evidence regarding its efficacy is equivocal. NSAIDS may improve pain and shorten recovery time, but concomitant renal failure often precludes their use, and thus opioids for severe pain may be indicated. In a recent systematic review, patients with diabetic muscle infarction who underwent surgery had a mean time to symptom resolution of $>80$ days, compared to around 40 days for patients with bed rest. This study also found that the recurrence rate was higher in patients who underwent surgery. ${ }^{9}$ Physicians should also be cautioned that physical therapy should be avoided until the lesion is fully healed, as this may also prolong recovery time.,

Although patients frequently recover from diabetic muscle infarction, this entity is a poor prognostic indicator of longterm clinical outcomes. Studies indicate that most patients suffer serious complications, including death, within five years of diagnosis. ${ }^{3}$ Another study cites a $10 \%$ mortality rate within 2 years of the event. ${ }^{10}$ Recurrence in the same or contralateral limb is not uncommon, sometimes within weeks of the initial ischemic episode. $^{3,4}$ 


\section{Conclusions}

Limb pain in patients with diabetes mellitus is a common complaint encountered by providers across multiple levels of care and various specialties. Because the differential diagnosis is extensive and includes several limb- and life-threatening conditions, urgent evaluation is critical. Although diabetic muscle infarction is a rare cause of acute muscular pain in diabetic patients, it must be considered, since optimal treatment can be dramatically different and because it confers such a high risk for diabetes-related morbidity and mortality. In these patients, physical therapy should be avoided until the lesion has healed, and tighter glycemic control is imperative.

\section{KEY POINTS}

1. Diabetic muscle infarction is an under-recognized microvascular complication of type 1 or type 2 diabetes mellitus.

2. Patients are usually insulin-dependent and have poorly controlled blood glucose levels. Often present are other microvascular complications, such as nephropathy, retinopathy, or neuropathy.

3. Patients typically present with acute-onset unilateral limb pain, although bilateral infarction can occur in approximately $30 \%$ of cases.

4. There are no specific laboratory findings for diabetic muscle infarction, but MRI can show increased signal in T2weighted images.

5. Tight glycemic control and limb rest are the mainstays of treatment.
ACKNOWLEDGMENTS: All authors have read the policies outlined by the journal.

Corresponding Author: David Rudy, MD; Department of Internal MedicineK521 Kentucky Clinic, 740 South Limestone Street, Lexington, KY 40536, USA (e-mail: dwrudy0@email.uky.edu).

Compliance with ethical standards:

Conflict of Interest: The authors declare that they do not have a conflict of interest.

\section{REFERENCES}

1. Trujillo-Santos AJ. Diabetic muscle infarction: an underdiagnosed complication of long-standing diabetes. Diabetes Care. 2003;26:2115

2. Choudhury BK, Saikia UK, Sarma D, Saikia M, Choudhury SD, Bhuyan D. Diabetic myonecrosis: an underreported complication of diabetes mellitus. Indian J Endocrinol Metab. 2011;15(1):58-61.

3. Rocca PV, Alloway JA, Nashel DJ. Diabetic muscular infarction. Semin Arthritis Rheum. 1993;22(4):280.

4. Umpierrez GE, Stiles RG, Kleinbart J, Krendel DA, Watts NB. Diabetic muscle infarction. Am J Med. 1996;101(3):245-50.

5. Drosos G. Pyomyositis. A literature review. Acta Orthop Belg. 2005;71:9-16.

6. Delaney-Sathy LO, Fessell DP, Jacobson JA, Hayes CW. Sonography of diabetic muscle infarction with MR imaging, CT, and pathologic correlation. AJR Am J Roentgenol. 2000;174(1): 165.

7. Morcuende JA, Dobbs MB, Buckwalter JA. Diabetic muscle infarction. Iowa Orthop J. 2000;20:65-74.

8. Grigoriadis E, Fam AG, Starok M, Ang LC. Skeletal muscle infarction in diabetes mellitus. J Rheumatol. 2000;27(4):1063-8.

9. Horton W, Taylor J, Ragland T, Subauste A. Diabetic muscle infarction: a systematic review. BMJ Open Diabetes Res Care. 2015;3(1):e000082.

10. Kapur S, Brunet JA, McKendry RJ. Diabetic muscle infarction: case report and review. J Rheumatol. 2004;31(1): 190.

11. Jelinek JS, Murphey MD, Aboulafia AJ, Dussault RG, Kaplan PA, Snearly WN. Muscle infarction in patients with diabetes mellitus: MR imaging findings. Radiology. 1999;211(1):241. 